\title{
Agentes causantes de Diarrea en Niños Menores de 5 Años en Tunja, Colombia
}

\author{
Fred G. Manrique-Abril ${ }^{\mathrm{I}}$, Diane Billon Y Tigne ${ }^{\mathrm{II}}$, Sandra E. Bello ${ }^{\mathrm{III}}$ y Juan M. Ospina ${ }^{\mathrm{IV}}$ \\ ${ }^{\text {I }}$ Enfermero. Ph. D. Salud Pública (candidato). M. Sc. Salud Pública. Escuela de Medicina-Enfermería \\ Universidad Pedagógica y Tecnológica de Colombia. E-mail: gisp@tunja.uptc.edu.co \\ II Fisioterapeuta. Especialista en Gerencia Hospitalaria. Investigadora Independiente Health Care \\ Colombia. E-mail: disabivi@yahoo.es \\ III Bacterióloga. Escuela de Medicina Universidad Pedagógica y Tecnológica de Colombia. E-mail: \\ sediith@yahoo.es \\ IV Médico. Escuela de Medicina Universidad Pedagógica y Tecnológica de Colombia. E-mail: \\ duendex56@yahoo.com
}

Recibido 18 Mayo 2005/Enviado para Modificación 16 Agosto 2005/Aceptado 15 Febrero 2006

\section{RESUMEN}

Objetivos Determinar la prevalencia y tipo de agentes infecciosos causantes de EDA en una muestra de niños menores de 5 años que consultaron a IPS de Tunja durante el año 2004.

Materiales y Métodos Estudio de corte transversal, la información se recogió mediante aplicación de una encuesta a 129 niños menores de 5 años afectados de EDA. Adicionalmente se recogió una muestra de heces, en las consultas externas del Hospital San Rafael y Clínica Saludcoop de Tunja.

Resultados Se encontró Rotavirus en 48,1 \%, Shigella 0,8 \%, E. coli 13,9 \%; Campylobacter 2,3 \%; Giardia lamblia 12,4 \%; E. histolytica $7 \%$; en 15,5 $\%$ de casos no se identificó agente causal. La asociación es creciente con la edad para Rotavirus $(\mathrm{p}<0,01)$, E.Coli $(\mathrm{p}<0,05)$ y Campylobacter $(\mathrm{p}<0,001)$.

Conclusión Rotavirus es el mayor agente causal de EDA en menores de 1 año, y en general, en menores de 5 años. Las prevalencias encontradas coinciden con lo reportado en estudios realizados en Facatativá, Bogotá, Santander, Manizales y Chocó; también con estudios adelantados en Venezuela, Perú y México.

Palabras Clave: Diarrea, rotavirus, salud pública, enfermedades transmisibles (fuente: DeCS, BIREME).

\section{ABSTRACT}

Diarrhoea-causing agents in children aged less than five in Tunja, Colombia 
Objectives Determining the prevalence and type of infectious ADD-causing agents in a sample of children aged less than five who consulted the IPS in Tunja during 2004.

Material and Methods A cross-sectional study was designed. Data was obtained by surveying 129 children younger than 5 suffering from ADD. Samples of faeces were obtained following outpatient consultation at San Rafael Hospital and SaludCoop's clinic in Tunja.

Results Rotavirus was found in $48,1 \%$ of cases, Shigella in $0,8 \%$, E. coli in $13,9 \%$, Campylobacter in 2,3 \%, Giardia lamblia in $12,4 \%$ and E. hitolytica in $7 \%$. The causative agent could not be identified in $15,5 \%$ of cases. The statistical association grew with age for Rotavirus $(p<0.01)$, E. coli $(p<0.05)$ and campylobacter $(\mathrm{p}<0.001)$.

Conclusion Rotavirus is the major causative agent of ADD in children younger than one year and, generally, in children aged less than five. The prevalence found was similar to data reported in studies carried out in Facatatativá, Bogotá, Santander, Manizales and the Chocó in Colombia and studies carried out in Venezuela, Peru and Mexico.

Key Words: Diarrhea, rotavirus, public health, communicable disease (source: MeSH, NLM).

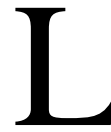

a diarrea constituye un problema de salud pública en el mundo, especialmente en países en desarrollo. Se estima que en Asia, África y América Latina, mueren cada año cerca de 3,3 millones de menores de 5 años por diarrea (1) y ocurren más de mil millones de episodios por año (2). Múltiples episodios de diarrea en el primer año pueden deteriorar el estado nutricional y generar graves secuelas (3-4).

En Colombia, la Enfermedad Diarreica aguda (EDA), ocupa los primeros lugares de morbimortalidad en población menor de cinco años, especialmente en los municipios menos desarrollados. Las tasas de mortalidad por EDA, han disminuido considerablemente, desde 225 a 31,7 por 100000 habitantes en el período 1981-1995. No obstante, no ha ocurrido lo mismo con la morbilidad, la tendencia es al aumento: de 110 a 113 casos por cada 1000 habitantes entre 1990 y 1996 (5), cifras similares a las registradas a escala mundial.

Las características epidemiológicas, agentes etiológicos y presentación clínica de la EDA varían dependiendo del País, región o comunidad, por lo que su reconocimiento en el ámbito local es útil en el diseño de programas de prevención y control, por ejemplo, la introducción de vacunas activas contra Rotavirus (5-9). En la actualidad, las investigaciones etiológicas de la 
EDA se inclinan hacia los estudios moleculares (9-11), no obstante, las técnicas convencionales de terreno siguen siendo una herramienta insustituible. El análisis microbiológico de las heces cobra valor en niños con cuadros diarreicos, en términos de la relación costo-beneficio; no obstante, en ocasiones el coprológico es útil para direccionar medidas de prevención de la diseminación de estos agentes, aún en ausencia de cuadros diarreicos, al permitir la identificación de fuentes ocultas en niños asintomáticos, que podrían representar riesgo potencial para las poblaciones (12).

Se propuso determinar la prevalencia de diferentes agentes infecciosos causantes de EDA en una muestra de lactantes y preescolares menores de 5 años, atendidos en instituciones prestadoras de salud (IPS), en Tunja, Boyacá, y evaluar la posible asociación de episodios de EDA con factores personales y sociodemográficos a los que están expuestos los niños, para tipificar un perfil detallado que permita orientar acciones concretas que disminuyan la morbilidad y eviten las complicaciones de la diarrea infecciosa.

\section{MATERIALES Y MÉTODOS}

Población: Entre el 1 de marzo y 30 de noviembre de 2004 se analizaron 129 casos de diarrea aguda en la consulta externa de 2 IPS de Tunja (Saludcoop, Hospital San Rafael) y cumplían con los siguientes criterios de inclusión: menor de 5 años, diagnostico de EDA al ingreso, no haber recibido antibióticos durante las tres semanas previas, no encontrarse en estado de choque ni gravemente desnutrido y no padecer alteraciones congénitas. La EDA se definió como presencia de tres o más evacuaciones líquidas o semilíquidas, o de una evacuación atípica y sanguinolenta, en 24 horas.

Previo consentimiento informado de los adultos acompañantes, se registró en el momento de la consulta: edad, sexo, procedencia; nivel socioeconómico, seguridad social de la familia, grado de instrucción y ocupación de la madre o responsable y presencia de mascotas, las manifestaciones clínicas: fiebre, vómitos, número de evacuaciones, deshidratación y duración de la diarrea, características de las heces: consistencia, presencia de sangre. En cada caso se recogió una muestra para coprocultivo por hisopado rectal, que se depositó en medio de transporte Cary Blair. Se tomó en caja de coprológico una muestra para los demás exámenes, Las muestras fueron procesadas en el Laboratorio de Microbiología de la Universidad Pedagógica y Tecnológica de Colombia (UPTC), máximo 6 horas después de la recolección.

Procesamiento de las muestras. La presencia de Rotavirus se determinó en muestras coprológicas, mediante test de aglutinación en látex con Anti- 
cuerpos monoclonales “Slider Rota-kit 2” ${ }^{\circledR}$ de laboratorios Biomerieux ${ }^{\circledR}$, siguiendo procedimientos ya estandarizados (13). La presencia de bacterias se estudió en muestras mantenidas a $4^{\circ} \mathrm{C}$ hasta su procesamiento, las que fueron sembradas en agar de McComkey, agar Salmonella-Shigella, agar xilosa-lisina-desoxicolato (XLD), y se realizaron pruebas bioquímicas para diferenciar especie, tales como, descarboxilación de la Lisina, producción de ácido sulfhidrico, fermentacion de azúcares, Indol, Voges proskauer, Simon Citrato, entre otros. Los parásitos Entamoeba histolytica y Giardia lamblia se investigaron en la hora siguiente a la obtención de la muestra, mediante examen en fresco de las heces por observación directa con solución salina 0,85 \% y con coloración de Lugol, mediante método de concentración de Kato-Kats. Se confirmó presencia de Entamoeba histolytica utilizando coloración de hematoxilina férrica (14).

Los datos fueron analizados en EPINFO 2002. Se estimaron diferencias entre las covariables y la variable de interés: agente etiológico de la EDA, mediante la determinación de la razón de prevalencias. Para la comparación los resultados entre variables cualitativas que se expresan en porcentajes, se calcularon los límites de confianza al 95 \% y se utilizó como prueba estadística Chí $^{2}$ y prueba exacta de Fisher Se consideraron resultados estadísticamente significativos si $\mathrm{p}<0,05$. o $\mathrm{p}<0,01$.

\section{RESULTADOS}

Durante los meses de marzo a Noviembre de 2004 se incluyeron 129 pacientes menores de 5 años con diarrea en los servicios de consulta externa, cumplieron con los criterios de inclusión, 51 niños (39,6 \%) del Hospital San Rafael de Tunja y 78 niños $(60,4 \%)$ de la Clínica Saludcoop. Los casos estudiados provenían en un 71,2 \% de la zona Urbana. Aunque el $90 \%$ de los usuarios proceden de Tunja o de municipios cercanos, algunos niños fueron remitidos por la red pública del departamento. Los datos se distribuyeron uniformemente por sexo, y edad (Tabla 1) sin diferencias estadísticas significativas $\mathrm{p}>0,05$; la edad promedio de los niños fue de 11,2 \pm 3 meses.

$\mathrm{Al}$ analizar la muestra se encuentra mayor afectación por EDA en los varones respecto de las mujeres, no se evidenciaron diferencias por edad ni por el grado de instrucción de la madre, hubo diferencias significativas con NBI $(p<0,01)$. Las características clínicas de la EDA en los pacientes estudiados se presentan en la Tabla 2, se evidencia que la deshidratación ocurre en 76,7 \% de los casos, al igual que anorexia (70,5\%). Una diferencia entre la proporción $\mathrm{p}<0,01$, de diarreas acuosas $60,5 \%$ con disentéricas 49,5\%. 
Se logro en un 83,1 \% de casos establecer el agente causal, porcentaje relativamente alto, a pesar de usar métodos tradicionales y de bajo costo, en el 16,88 \% no se logró identificar agente etiológico. El agente detectado con mayor frecuencia fue Rotavirus, (Tabla 2).

Tabla 1. Características generales de los pacientes

\begin{tabular}{|c|c|c|c|}
\hline \multicolumn{2}{|c|}{ Característica } & \multirow{2}{*}{$\begin{array}{c}\text { Número } \\
77\end{array}$} & \multirow{2}{*}{$\begin{array}{c}\% \\
59,7\end{array}$} \\
\hline Sexo & Masculino & & \\
\hline & Femenino & 52 & 40,3 \\
\hline \multirow[t]{4}{*}{ Edad } & 0 a 6 meses & 49 & 38,0 \\
\hline & 6 a 12 meses & 33 & 25,6 \\
\hline & 12 a 36 meses & 27 & 20,9 \\
\hline & 36 y mas & 20 & 15,5 \\
\hline \multirow[t]{2}{*}{ Institución de salud } & HSRT & 51 & 39,5 \\
\hline & SALUDCOOP & 78 & 60,5 \\
\hline \multirow{4}{*}{$\begin{array}{l}\text { Grado de nstrucción } \\
\text { de la madre o } \\
\text { responsable del } \\
\text { niño }\end{array}$} & Ninguna & 6 & 4,6 \\
\hline & Primaria & 29 & 22,5 \\
\hline & Bachiller & 74 & 57,4 \\
\hline & Universitario & 20 & 15,5 \\
\hline \multirow{3}{*}{$\begin{array}{l}\text { Necesidades } \\
\text { básicas } \\
\text { Insatisfechas. NBI }\end{array}$} & Pobreza & 34 & 26,4 \\
\hline & Miseria & 7 & 5,4 \\
\hline & Normal & 88 & 68,2 \\
\hline
\end{tabular}

No hubo diferencias en la prevalencia por sexo $(p>0,05)$. La muestra se dividió en grupos por edad: menor de 6 meses, de 6 a 12 meses, 12 a 36 meses y mayor de 36 meses. Se encontraron diferencias para cada grupo de edad $(\mathrm{p}<0,01)$, con gradiente lineal para Rotavirus, E. coli y Giardia y mas débil para E. Histolytica. La frecuencia de Rotavirus disminuyó con la edad $(\mathrm{p}<0,01)$, en contraste, $E$. coli aumenta en frecuencia a medida que aumenta la edad ( $<<0,01)$. Estas tendencias se encontraron estadísticamente homogéneas, (valor p test homogeneidad: $<0,01$ ), el efecto en E. histolytica es confuso, posiblemente por el tamaño de muestra. No se encontraron diferencias asociadas a edad en los otros microorganismos estudiados 


\section{DISCUSION}

Aunque se han adelantado evaluaciones similares en otras regiones de Colombia, en Boyacá es desconocida la prevalencia de Rotavirus como agente causal de EDA. Estudios realizados en servicios de consulta externa y hospitalización en Facatativa (16), Bogotá (5,16,20), Medellín (21-22), Manizales y Barranquilla (23-24), que tuvieron también similar tiempo de seguimiento en Medellín (16,21), Bogotá (19) y Pereira (22), reportaron frecuencias similares para agentes etiológicos causantes de EDA. Una limitante importante es la imposibilidad para analizar estacionalidad y relaciones de la EDA con condiciones climáticas y ambientales.

Tabla 2. Características clínicas de los pacientes y tipo de microorganismos identificados

\begin{tabular}{|c|c|c|c|c|}
\hline \multicolumn{2}{|c|}{ Característica } & Número & $\%$ & IC 95\% (15) \\
\hline \multirow[t]{3}{*}{ Deshidratación (DHT) } & Sin DHT & 30 & 23,3 & $(15,6-30,9)$ \\
\hline & Leve & 84 & 65,1 & $(56,5-73,2)$ \\
\hline & Moderada & 15 & 11,6 & $(5,7-17,5)$ \\
\hline \multirow[t]{3}{*}{ No de deposiciones } & $<3$ & 52 & 40,3 & $(31,5-49,2)$ \\
\hline & 3 a 5 & 51 & 39,5 & $(30,7-48,4)$ \\
\hline & $>5$ & 26 & 20,2 & $(12,9-27,5)$ \\
\hline Anorexia & $\mathrm{Si}$ & 91 & 70,5 & $(62,3-78,8)$ \\
\hline Fiebre & $\mathrm{Si}$ & 25 & 19,4 & $(12,2-26,6)$ \\
\hline Vómitos & $\mathrm{Si}$ & 32 & 24,8 & $(16,9-32,6)$ \\
\hline \multirow[t]{3}{*}{ Desnutrición (DNT ) } & Crónica & 25 & 19,4 & $(12,2-26,6)$ \\
\hline & Global & 32 & 24,8 & $(16,9-32,6)$ \\
\hline & Aguda & 27 & 20,9 & $(13,5-28,3)$ \\
\hline \multirow[t]{5}{*}{ Tipo de diarrea } & Acuosa & 78 & 60,5 & $(51,6-69,3)$ \\
\hline & Disenterica & 51 & 39,5 & $(30,7-48,4)$ \\
\hline & Rotavirus & 62 & 48,1 & $(39,3-56,9)$ \\
\hline & Shígella & 1 & 1,3 & $(0,0-4,9)$ \\
\hline & E. coli & 18 & 13,9 & $(8,7-21,4)$ \\
\hline \multirow{4}{*}{$\begin{array}{l}\text { Microorganismo } \\
\text { identificado }\end{array}$} & Campylobacter & 3 & 2,3 & $(0,6-7,2)$ \\
\hline & Giardia lamblia & 16 & 12,4 & $(7,5-19,6)$ \\
\hline & E. Histolytica & 9 & 6,9 & $(3,4-13,2)$ \\
\hline & Ninguno & 13 & 16,8 & $(9,9-23,2)$ \\
\hline
\end{tabular}

El estudio permite una aproximación a la etiología de la EDA en Boyacá, lo que repercutirá en mejor planificación y prevención de las entidades actorasde la política sanitaria: el Instituto de Salud de Boyacá. Los resultados confirman la hipótesis que identifica al Rotavirus como primer agente etiológico de EDA; se puede considerar que entre el 39 y $56 \%$ de las diarreas que ocurren en menores de cinco años, en la capital del departamento sean 
causadas por Rotavirus. Por esta razón, es importante fortalecer los sistemas de vigilancia para EDA y propiciar análisis de la carga de la enfermedad por Rotavirus con pruebas de aglutinación para virus, disponibles en el mercado, permitiendo mejorar la gama diagnóstica y el enfoque terapéutico.

Tabla 3. Prevalencia de agentes etiológicos por edad

\begin{tabular}{|c|c|c|c|c|c|c|}
\hline & & $<6$ meses & 6-12 meses & $12-36$ meses & $\begin{array}{l}>36 \\
\text { meses }\end{array}$ & Total \\
\hline \multirow[t]{2}{*}{ Rotavirus } & No. & 36 & 18 & 6 & 2 & 62 \\
\hline & Prev & 0,73 & 0,54 & 0,22 & 0,1 & 0,48 \\
\hline \multirow[t]{2}{*}{ Shigella } & No. & 0 & 0 & 0 & 1 & 1 \\
\hline & Prev & - & - & - & 0 & 0 \\
\hline \multirow[t]{2}{*}{ E. Coli } & No. & 1 & 4 & 5 & 8 & 18 \\
\hline & Prev & 0,02 & 0,12 & 0,18 & 0,4 & 0,14 \\
\hline \multirow[t]{2}{*}{ Campylobacter } & No. & 2 & 0 & 1 & 0 & 3 \\
\hline & Prev & 0.04 & 0 & 0.03 & 0 & 0,02 \\
\hline \multirow[t]{2}{*}{ Giardia } & No. & 1 & 3 & 5 & 7 & 16 \\
\hline & Prev & 0,02 & 0,09 & 0,18 & 0,35 & 0,12 \\
\hline \multirow[t]{2}{*}{ E. Histolytica } & No. & 0 & 3 & 4 & 2 & 9 \\
\hline & Prev & 0 & 0,09 & 0,14 & 0,1 & 0,07 \\
\hline Desconocido & $\begin{array}{l}\text { No. } \\
\text { Prev }\end{array}$ & $\begin{array}{c}9 \\
0,18 \\
\end{array}$ & $\begin{array}{c}5 \\
0,15 \\
\end{array}$ & $\begin{array}{c}6 \\
0,22 \\
\end{array}$ & $\begin{array}{l}0 \\
0\end{array}$ & $\begin{array}{c}20 \\
0,15 \\
\end{array}$ \\
\hline
\end{tabular}

En estudios realizados en Colombia (5), se reportó $57 \%$ de pruebas positivas para Rotavirus en niños de hasta 59 meses. Se encuentra que $70 \%$ de pruebas positivas para Rotavirus ocurren en menores de 24 meses. Los grupos de edad más afectados son: 6 a 11 meses 33,7 \%, seguidos por niños de 12 a 23 meses 30,9\%. De 24 meses en adelante, el porcentaje de pruebas positivas es menor, 24 a 35 meses: 12,7 \%; 36 a 47 meses: 9,1 \% y 48 a 59 meses: $6,5 \%$.

Se concluye que Rotavirus debe ser considerado como principal agente patógeno causante de diarrea en menores de 5 años, con clínica marcada por: diarrea acuosa, deshidratación, vómitos y fiebre ocasional, cuadro que coincide con la clínica consignada en los protocolos internacionales. (24-26).

La frecuencia de Shigella como agente causal sugiere la necesidad de hacer controles de vigilancia ambiental por parte del municipio. Algunos autores consideran que Salmonella es un agente importante en niños (5,2426), Los resultados coinciden con otros reportes que han encontrado baja frecuencia de esta bacteria como agente etiológico de diarrea en niños $(5,16,27-33)$. 
La edad parece ser factor importante de prevalencia y gravedad de la diarrea, con marcada diferencia para Rotavirus y bacterias. También encontramos que un bajo nivel educativo, tenencia de mascotas y deficiencias higiénicas en la vivienda se asocian con presencia de agentes etiológicos causantes de diarrea.

Los resultados sugieren la conveniencia de desarrollar programas de promoción y prevención desde el nivel local. Las políticas generales se deberían dirigir desde los ámbitos regional y nacional, apoyando investigaciones que optimicen la interacción Universidad - Entes generadores de políticas en salud, mejorando las perspectivas de prevención con el desarrollo de vacunas o métodos profilácticos efectivos sobre todo en Colombia, donde son limitados los recursos sociales y financieros para la investigación en salud e implementación de programas de control •

Agradecimientos. A la Dirección de Investigaciones de la UPTC y al Instituto Seccional de Salud de Boyacá. A los funcionarios de la Clínica Saludcoop y Hospital San Rafael. A los estudiantes Diego Alexander Manrique, Marlon Fernando Tejedor B, Lina Fernanda Barrera S. y Miguel Ángel Pineda R. de la UPTC por su colaboración en la recolección de muestras.

\section{REFERENCIAS}

1. Bern C, Martinez J, De Zoisa I, Glass RI. The magnitude of the global problem of diarrheal disease: a ten-year update. Bull World Health Organ 1992;70 (6):705-714.

2. Bern C, Glass RI. Impact of diarrheal disease worlwide. In: Kapikian AZ, ed. Viral infections of the gastrointestinal tract. 2 Ed. New York: Marcel Dekker; 1994. pp. 1-26.

3. Guerrant RL, Schorling JB, McAuliffe JF, De Souza MA. Diarrhea as a cause and effect of malnutrition: diarrhea prevents catch-up growth and malnutrition increases diarrhea frequency and duration. Am J Trop Med Hyg 1992;47 (1):28-35.

4. Perez-Schael I, Dehollain P, Perez M, Daoud N, Soto I, Gonzalez M, et al. Impacto de las enfermedades diarreicas en el estado nutricional del niño. An Ven Nutr 1988;1: 119-128.

5. Instituto Nacional de Salud. Inf Quinc Epidemiol Nac 2003;8(20):321-36.

6. Ministerio de Salud. Normas Técnicas y Guías De Atención Tomo II. Resolución. Número 0412 de febrero 25 de 2000. Bogotá DC; 2000.

7. Kapikian AZ, Hoshino Y, Chanock RM, Perez-Schael I. Efficacy of a quadrivalent rhesus rotavirus-based human rotavirus vaccine aimed at preventing severe rotavirus diarrhea in infants and young children. J Infect Dis 1996;174(Supl 1):S65-S72. 
8. Perez-Schael I, Guntiñas MJ, Perez M, Pagone V, Rojas AM, Gonzalez R, et al. Efficacy of rhesus rotavirus-based uadrivalent vaccine in infants and young children in Venezuela. N Engl J Med 1997;337(17):1181-1187.

9. Glass RI, Bresee JS, Parashar U, Miller M, Gentsch JR. Rotavirus vaccines at the threshold. Nat Med 1997;3(12):1324-1325.

10. Correa A, Solarte Y, Barrera J, Mogollón D, Gutiérrez MF. Caracterización molecular de rotavirus en la ciudad de Santafé de Bogotá, Colombia. Determinación de electroferotipos y tipificación de una cepa por RT-PCR. Rev Latinoam Microbiol. 1999;41 (3):167-73.

11. Bermeo L.A., Mogollón J.D, Gutiérrez M.F., Ariza F, Barrera J-D, Jerabek l. Molecular characterization of rotavirus strains obtained from human diarrheic samples and their epidemiological implications. Universitas Scientiarum 1997; 4(1):71-81.

12. Organización Mundial de la Salud. Métodos básicos de laboratorio en bacteriología clínica. Ginebra; 1993. pp. 37-8.

13. Gutiérrez MF, Figueroa S, Torres C. Valor del examen coprológico y coproscópico en la infección por Rotavirus. Laboratorio \& Medicina. 1993; 1:5-9.

14. Arango A.E, Gutiérrez M.F. Diagnóstico clásico de las infecciones virales. En: Principios de Virología. Ediciones Gráficas Ltda. 2000. Medellín, Colombia.

15. Fleiss JL, statistical methods for rates and proportions, 2nd editions, John Wiley y Sans. 1981, pp-14-15.

16. Gutiérrez MF, Valencia C., Bermeo l., Mogollón D., Barrera J.D, Ariza F. Determinación de Rotavirus humanos y porcinos en algunas ciudades colombianas: Evaluación de técnicas diagnósticas. Laboratorio Actual 1999.16(32):3-9.

17. Gutiérrez MF, Serrano P, Vanegas C, Macias A., Riaño M. Efecto de las variaciones climáticas en gastroenteritis causadas por Rotavirus y Adenovirus en niños menores de cuatro años en Santafé de Bogotá entre junio de 1996 y junio de 1998. MEDICAS UIS 2000; 14:24-29.

18. Mora JO, Juliao O, Suescún J, Guzmán M. Estudio longitudinal sobre la epidemiología y la etiología de la enfermedad diarreica aguda en los niños de una comunidad urbana pobre de Bogotá, Colombia. Instituto Nacional de Salud. Publicaciones Científicas; № 15. Bogotá, 1988

19. Leal FJ, Franco G, Sandoval C, Gómez E, Buitrago B, Rodríguez G, et al. Agentes etiológicos de diarrea aguda en Bogotá. Santa Fé de Bogotá: Federación Colombiana de Especialistas en Laboratorio Clínico (FE-CODEL); 1984. pp. 5-55.

20. Guerrero C. Caracterización electroforética de rotavirus aislados de infantes con gastroenteritis en la ciudad de Bogotá. Rev Fac Med UN 1994; 42(4):203208.

21. Arango AE, Aguirre C, Aristizábal L, Hanssen H. Frecuencia de rotavirus en niños menores de cinco años con diarrea aguda. Primer informe. Estudio en un grupo de niños de la ciudad de Medellín. Salud Uninorte 1985; 2(3): 129-138. 
22. Trujillo H, Jaramillo C, Restrepo M., Mejía de R. GI, Zapata CM., Ramírez R. Rotavirus y otros enteropatógenos en la etiología de la diarrea aguda en Medellín, Colombia 1982; Bol Of Sanit Panam 1985: 98(3), 251-259

23. Remolina A, Rey F, de García L, Llanos R, Arellana R. Clemow R, Palacio N, Fals E. Etiología de la enfermedad diarreica aguda (EDA.) en Barranquilla, Colombia. Salud Uninorte 1986; 3: 3-18.

24. Remolina A, Rey F, de García L, Llanos R, ArellanaR. Clemow R, Palacio N, Fals E. La enfermedad diarreica aguda (EDA): estudios epidemiológicos en el departamento del Atlántico, Colombia, Salud Uninorte 1985; 2:(2)71-79.

25. Urbina D, Arzuza O, Young G, Parra E, Castro R, Puello M. Rotavirus type A and other enteric pathogens in stool samples from children with acute diarrhea on the Colombian northern coast. Int Microbiol. 2003; 6(1):27-32.

26. Black RE, Merson MH, Rahman AS, Yunus M, Alim AR, Huq I, et al. A twoyear study of bacterial, viral, and parasitic agents associated with diarrhea in rural Bangladesh. J Infect Dis 1980;142(5):660-664.

27. Echeverria P, Taylor DN, Lexsomboon U, Bhaibulaya M, Blacklow NR, Tamura $\mathrm{K}$, et al. Case-control study of endemic diarrheal disease in Thai children. J Infect Dis 1989;159(3): 543-548.

28. Huilan S, Zhen LG, Mathan MM, Mathew MM, Olarte J, Espejo R, et al. Etiology of acute diarrhoea in developing countries: a multicentre study in five countries. Bull World Health Organ 1991;69(5):549-555.

29. Seigel RR, Santana C, Salgado K, De Jesus P. Acute diarrhea among children from high and low socioeconomic communities in Salvador, Brazil. Int J Infect Dis 1996;1(1):28-34.

30. Varavithya W, Vathanophas K, Bodhidatta L, Punyaratabandhu P, Sangchai R, Athipanyakom S, et al. Importance of Salmonellae and Campylobacter jejuni in the etiology of diarrheal disease among children less than 5 years of age in a community in Bangkok, Thailand. J Clin Microbiol 990;28(11):2507-2510.

31. Gomes TAT, Rassi V, MacDonald KL, Ramos SR, Trabulsi LR, Vieira MAM, et al. Enteropathogens associated with acute diarrheal disease in urban infants in Sao Paulo, Brazil. J Infect Dis 1991;164(2):331-337.

32. Molbak K, Wested N, Holyng N, Scheutz F, Gottschau A, Aaby P, et al. The etiology of early childhood diarrhea: a community study from Guinea-Bissau. J Infect Dis 1994;169(3): 581-587.

33. Ogunsanya TI, Rotimi VO, Adenuga A. A study of the etiological agents of childhood diarrhea in Lagos, Nigeria. J Med Microbiol 1994;40(1):10-14.

34. Urrestarazu M, Liprandi F, Perez E, Gonzalez R, Perez I. Características etiológicas, clínicas y sociodemográficas de la diarrea aguda en Venezuela. Rev Panam Salud Publica. 1999; 6(3) 149-156. 